\title{
EMISSION-LIKE FEATURE DUE TO A LATITUDINAL DIFFERENTIAL ROTATION
}

\author{
J. ZOREC \\ Institut d'Astrophysique de Paris - 98 ${ }^{b i s}$, Bd. Arago, 75014 Paris, France
}

\begin{abstract}
We simulate the effect due to a latitudinal differential rotation on a $\mathrm{HeI} \lambda \mathbf{\lambda 4 7 1}$ absorption line. The effect can produce an emission-like reversal in the line core.
\end{abstract}

\section{Introduction}

Central emission-like reversals in some absorption lines of a number of Btype stars have been reported by Baade (1990). Baade (1990) and Jeffery (1991) studied the possibility of producing this emission through the gravitational darkening effect. In this paper we present a simple geometrical effect related to a latitudinal differential rotation on the stellar surface which can also be responsible for shallow absorption profiles or even for emission-like reversals.

\section{The model}

To clearly distinguish this effect from that discussed by Baade (1990) and Jeffery (1991), we suppose that the effective temperature and gravity are uniform over the stellar surface and we neglect any stellar geometrical deformation. The simulation of a latitudinal differential rotation is performed using a conservative angular velocity law $\Omega(\varpi) \propto \varpi^{-2}\left[1-\left(1-m_{\varpi}\right)^{2 / 3}\right]\left(\mathrm{m}_{\varpi}\right.$ is the fractional stellar mass inside a cylinder of radius $\varpi$ ) which results when a star contracts conserving angular momentum by cylindrical shells. Note that rapidly rotating early-type stars may look like Jupiter (Zahn 1983). For laws of the type $\Omega=\Omega(\varpi)$, lines of constant radial velocity $\mathcal{C} \equiv \Omega(\varpi) \eta \sin$ i $=$ const. $(\eta$ is the horizontal coordinate in the stellar disc projected on the sky and perpendicular to the line of sight) are not straight ones as for rigid rotation $(\Omega=$ constant), but they are curved ones, and some of them are closed (see Fig. 1a). Also, the maximum radial velocity is not on the limb at the equator, but at a point where $\partial[\Omega(\varpi) \eta] \partial \eta=0$.

\section{The effect}

Classical rotational broadening of a spectral line results as a consequence of a Doppler displacement, where the contribution to the absorption at each wavelength is proportional to the length of the respective line of constant radial velocity on the projected stellar disc. Our effect follows the same logic, 
but the length of $\mathcal{C}$ curves varies from a relative maximum at the centre of the stellar disc (which produces the central emission), passes through an absolute maximum (which produces the maximum absorption), and then, decreases toward zero where $[(\Omega(\varpi) \eta \sin i)]_{M a x}=\mathrm{V} \sin$ i. An example of rotationally broadened profile of $\mathrm{HeI} \lambda 4471$ for $\mathrm{V} \sin \mathrm{i}=100 \mathrm{~km} \mathrm{~s}^{-1}\left(\mathrm{~T}_{\text {eff }}=\right.$ $20000 \mathrm{~K}, \log \mathrm{g}=4.0$ ) is shown in Fig. 1b. Allowing for the gravity darkening effect, the emission-like reversal can be more or less contrasted depending on the sensitivity of the line to the temperature and to the gravity. It is expected that time dependent $\Omega(\varpi, \mathrm{t})$ laws will introduce variable emissionlike components. Such an effect may also be due to superimposed horizontal currents in the stellar surface. When the ratio $\tau=K /|W|$ (K : rotational kinetic energy; W: gravitational potential energy) is $\tau_{c}^{R} \leq \tau \leq 0.10\left(\tau_{c}^{R}\right.$ : ratio for critical rigid rotation $\leq 0.008$ ) the strong polar deformation of the star introduces a double-valued problem for the $\mathrm{V}$ sin i parameter (Zorec et al. 1988).
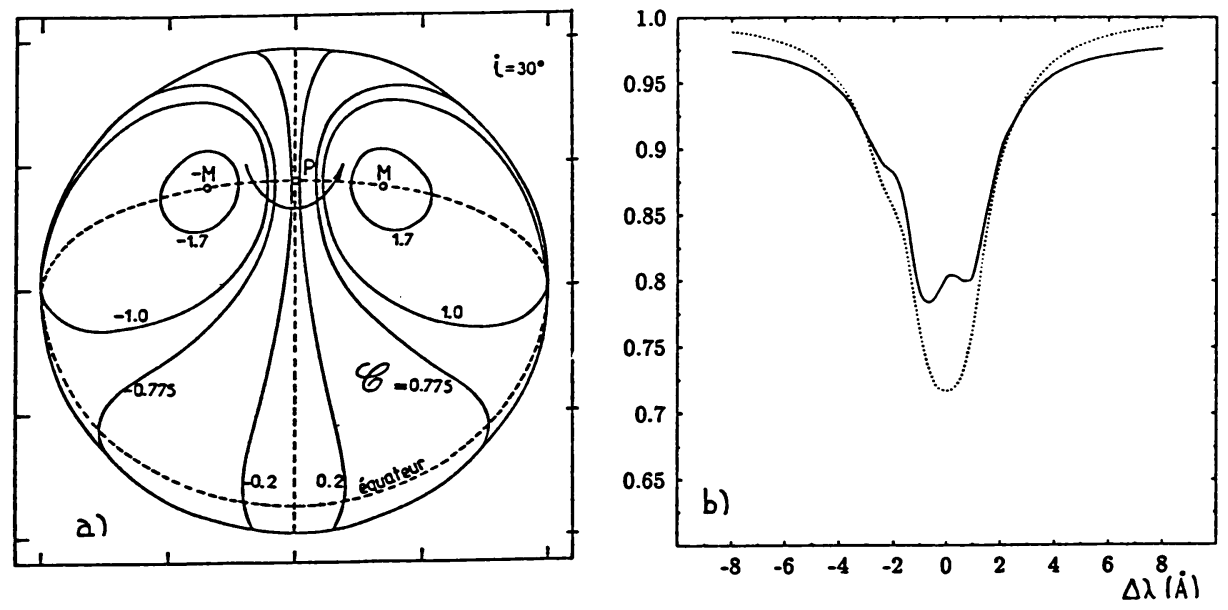

Fig. 1. a) Curves $\mathcal{C}$ of equal radial velocity at $i=30^{\circ} . \mathbf{P}:$ stellar pole; $\pm \mathbf{M}$ points of radial velocities $\pm \mathrm{V}_{\text {Max }} \sin \mathrm{i}$. b) (-): broadened profile of the HeI $\lambda 4471$ line for $\mathrm{V}_{\text {Max }}$ $\sin i=100 \mathrm{~km} \mathrm{~s}^{-1} ;(. .$.$) : profile broadened by a rigid rotation and same \mathrm{V}$ sin $\mathrm{i}$.

\section{References}

Baade, D.: 1990, in L.A. Wilson and R. Stalio, ed(s)., Angular Momentum and Mass Loss for Hot Stars, Kluwer Acad. Publ., 177

Jeffery, C.S.: 1991, Mon. Not. R. astr. Soc. 249, 327

Zahn, J.-P.: 1983, Astroph. Processes in Upper Main Seq. Stars, Obs. Genève, 253

Zorec, J., Mochkovitch, R., Garcia, A.: 1988, C.R. Acad. Sci. Paris 306, 1225 